\title{
SIMPLIFIED ANALYTICAL METHODS TO ANALYZE LOCK GATES SUBMITTED TO SHIP COLLISIONS AND EARTHQUAKES
}

\author{
BULDGEN LOIC - Research Engineer, PhD, University of Liège, Faculty of Applied Sciences, ANAST \\ Department, e-mail: L.Buldgen@ulg.ac.be \\ BELA ANDREEA - PhD Student, University of Liège, Faculty of Applied Sciences, ANAST Department, \\ e-mail: a.bela@ulg.ac.be \\ RIGO PHILIPPE - Professor, University of Liège, Faculty of Applied Sciences, ANAST Department, \\ e-mail: ph.rigo@ulg.ac.be
}

\begin{abstract}
This paper presents two simplified analytical methods to analyze lock gates submitted to two different accidental loads. The case of an impact involving a vessel is first investigated. In this situation, the resistance of the struck gate is evaluated by assuming a local and a global deforming mode. The super-element method is used in the first case, while an equivalent beam model is simultaneously introduced to capture the overall bending motion of the structure. The second accidental load considered in this paper is the seismic action, for which an analytical method is presented to evaluate the total hydrodynamic pressure applied on a lock gate during an earthquake, due account being taken of the fluid-structure interaction. For each of these two actions, numerical validations are presented and the analytical results are compared to finite-element solutions.
\end{abstract}

Keywords: super-element method, local mode, global mode, hydrodynamic pressure, fluidstructure interaction.

\section{Introduction}

It is a fact that the freight transport demand is constantly growing since 1995 . Nowadays, $76 \%$ of the European goods transit by road and only $6 \%$ by water, which is apparently not viable because the road capacity is progressively reaching a maximum. The modal split has therefore to be changed, and a serious alternative is to develop the river transport. This choice can be motivated by environmental, economic and safety reasons, which justifies that the waterborne transport is a credible alternative to road transport. This is particularly true in Europe, where the five biggest seaports are connected to more than 37000 kilometers of navigable inland waterways. From these observations, the European Commission has precisely decided to promote the waterborne transport through the NAIADES I and II action programs. Amongst all the measures suggested by these plans, one of them consists in building new locks or improving the existing ones.

Of course, a direct consequence of such policies should be an increase of the waterborne traffic in the upcoming years. This means that the number of vessels passing though locks will be more important, which is also true for potential accidents. Therefore, in order to keep a sufficient level of safety, locks have to be properly designed. To achieve this goal, engineers have to investigate more carefully the effect of accidental actions, which is also a requirement from most international standards. Amongst all the loads that may be considered for the design of lock gates, ship impacts and earthquakes are the most difficult ones to handle because they are not really documented in the literature. Furthermore, the use of finite-element software is often needed to assess correctly their effects, but working with numerical tools is not always possible in the predesign stage because of time and cost limitations. Indeed, building finite-element models, running simulations and post-processing the results may be a quite long procedure.

From the previous considerations, it appears that an efficient tool is missing for integrating the seismic action and ship collisions in the pre-design of lock gates. The aim of this paper is precisely to present analytical methods that could be advantageously used to overcome these difficulties. 


\section{Ship collisions on lock gates}

\subsection{Deformation sequence}

In the simplified approach developed to analyze ship collisions on lock gates, it is assumed that the striking vessel is perfectly rigid, which implies that its initial kinetic energy has to be entirely dissipated by the deformation of the gate. Such an assumption is rather conservative, as in reality; the ship will also be affected during the collision. In order to establish an analytical procedure providing a quick evaluation of the collision resistanceP $(\delta)$, it is assumed that the gate may deform in the two following manners during the impact:

- the local deforming mode, which is associated to a localized indentation of the striking vessel. During this process, the major part of the structure is not affected by the impact and the penetration of the vessel is mainly allowed by the crushing of some elements in a confined area.

- the global deforming mode, which is associated to an overall deformation of the gate. In this case, the striking vessel keeps moving forward by involving displacements on the entire gate.

These concepts are roughly illustrated on Fig. 1, which shows the deflection of a plane lock gate. From this picture, it can be seen that a local indentation (1) is imposed near the impact point $I$, which may be directly related to the local deforming mode.

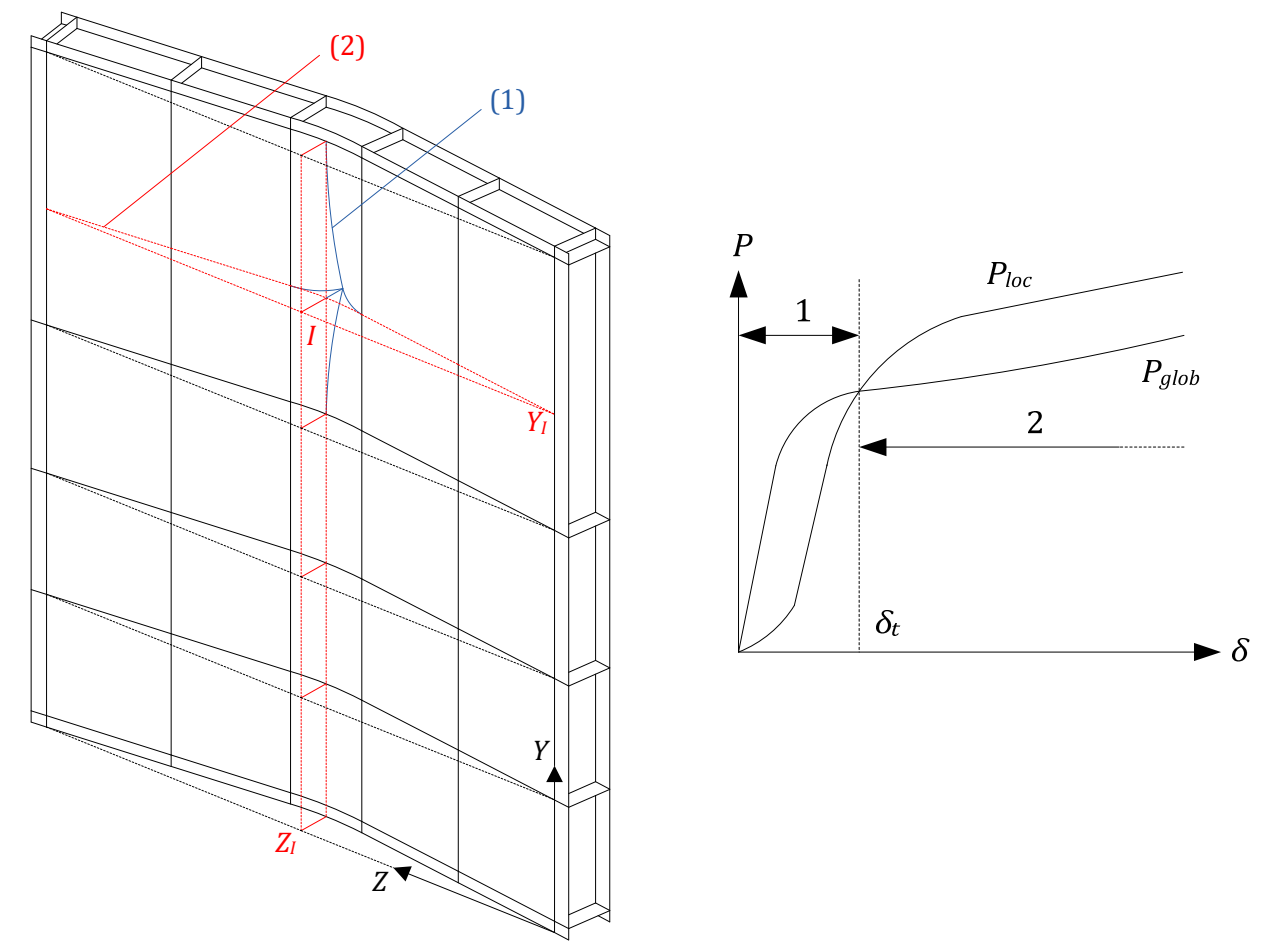

Fig. 1 - Global and local deforming modes

In addition, Fig. 1 also shows that an overall bending motion (2) of the entire structure is also activated, which may be associated to the global sequence. It is important to distinguish between these two modes, as they influence the behavior of the gate. More details about these phenomena may be found in references [1] to [4].

At the beginning of the collision process, it is clear that the gate will be affected in a quite small region, which means that the local deforming mode is first activated. Nevertheless, a larger part of the structure will be progressively involved, which implies that there should be a switch from the local to the global behavior. It is therefore required to detect when this transition is likely to take place. 
To do so, for a given penetration $\delta$ of the striking vessel, it can first be assumed that the local mode is activated, which allows to calculate the resistance $P_{l o c}$ of the gate. On the other hand, it also possible to imagine that the gate withstands through a global mode, for which another value $P_{\text {glob }}$ of the resistance may be derived. The basic idea to combine these two calculations is to assume that the total impact resistance $P$ is always minimal, which means:

$$
\begin{aligned}
& \text { - I } \\
& \text { - } \mathrm{f} P_{l o c}<P_{\text {glob }}: \quad P=P_{l o c} \quad \delta<\delta_{t} \text { : local deforming mode } \\
& \text { - If } P_{l o c}=P_{\text {glob }}: \quad P=P_{l o c} \quad \delta=\delta_{t} \text { : transition } \\
& \text { - } \quad \text { If } P_{l o c}>P_{\text {glob }}: \quad P=P_{g l o b} \quad \delta>\delta_{t} \text { : global deforming mode }
\end{aligned}
$$

where $\delta_{t}$ is the particular value of the penetration $\delta$ corresponding to the transition between the local and global behaviors. This procedure is presented on Fig. 1, from which it transpires that the analytical derivation of $P$ may be achieved by solving two different problems consisting in finding coherent closed-form solutions for $P_{l o c}$ and $P_{g l o b}$.

\subsection{Local resistance}

To calculate the resistance $P_{l o c}$ associated to the local deforming mode, the idea is to devide the gate into a set of $N$ large structural entities that are called super-elements. Each of them is characterized by a law giving its individual resistance $P_{i}$ as a function of the penetration $\delta$. As the present paper is only concerned with simple configurations having a single plating reinforced by an orthogonal set of horizontal girders and vertical frames (such as mitre or lifting gates for example), four different types of super-elements are required to decompose this type of structures into large entities. They are briefly described hereafter and depicted in Fig. 2a:

- Type A: this type of super-element is used to represent plating elements bounded by two vertical frames and two horizontal girders. They are modeled as a simply supported plate submitted to an out-of-plane impact load. In this case, the resistance is mainly provided through the development of tensile membrane forces.

- Type B: this type of super-element is used to model portions of vertical frames limited by two horizontal ones. For such elements, it is worth noting that the collision may not appear on one of their intersection. In other words, they are always impacted somewhere between the two delimiting horizontal girders (otherwise they have to be treated as a type C). During the collision, these components are submitted to a successive folding process.

- Type $C$ : this type of super-element is introduced to treat impacts occurring on the intersection between horizontal and vertical girders. They are supposed to be crushed axially, which also requires the development of successive folds.

- Type D: type D is the same as type B but this time it is applicable to portions of horizontal girders bounded by two vertical frames. Here again, the impact may not take place on their intersections, otherwise they have to be considered as a type C.

For each of the four different types introduced above, it is possible to find closed-form expressions relating the individual resistance of the super-element to the indentation. This can be achieved by applying the upper-bound theorem. To start this derivation, it is first required to postulate a kinematically admissible displacement field that represents more or less the behavior of the super-element during the impact. For example, one can imagine that the portions of the horizontal girders (type D) are submitted to the folding process of Fig. 2b during the collision. 
Once a compatible mechanism has been defined, then the next step is to evaluate the associated internal energy $\dot{E}_{i}$. According to the virtual work principle, the latter has to be equal to the power developed by the external force, i.e.:

$$
P_{i} \dot{\delta}=\dot{E}_{i} \Leftrightarrow P_{i}=\dot{E}_{i} / \dot{\delta}
$$

where $P_{i}$ is the resistance associated to the super-element $n^{\circ} i$ and $\dot{\delta}$ is the striking velocity. By applying equation (2) to the four types mentioned here above, it is possible to find closed-form expressions in each case. As this operation is quite fastidious, it is not reported here, but additional information on this topic may be found in the literature (see references [5] to [10] for example).

(a) The four different types of superelements

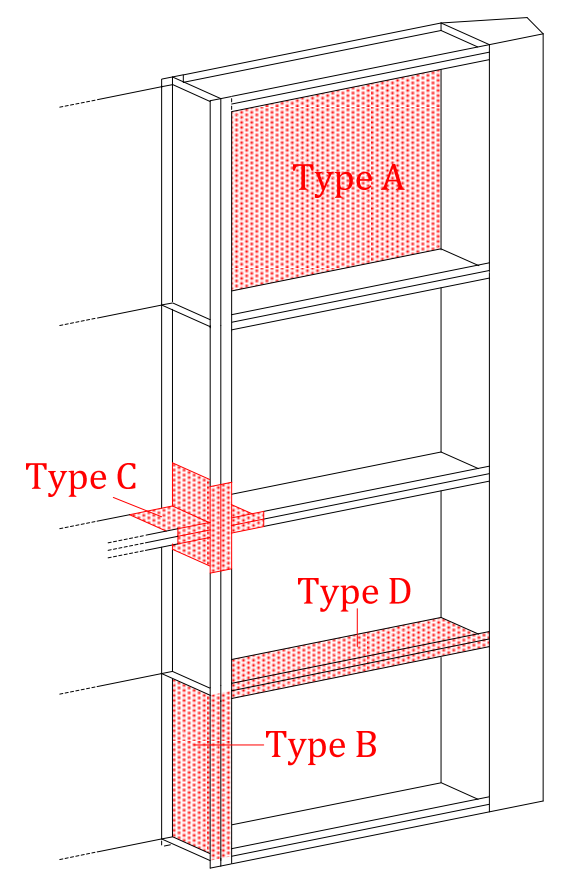

(b) Example of kinematically admissible mechanism for a superelement of type D

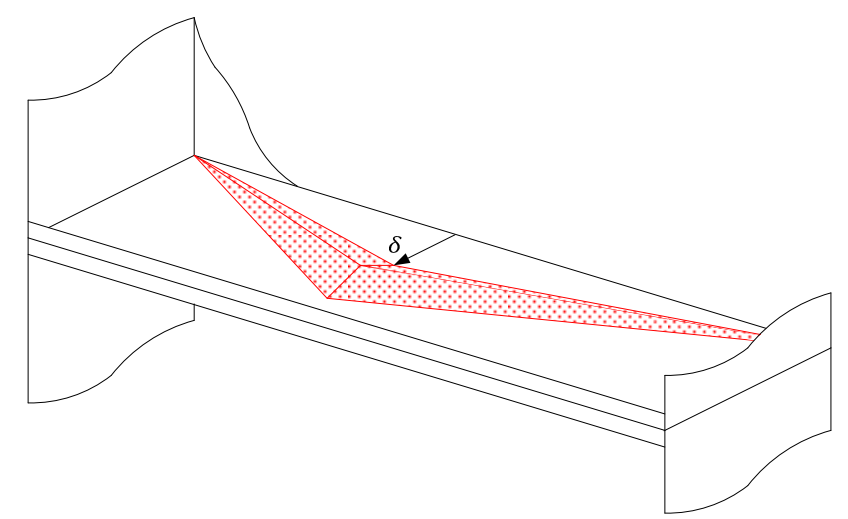

Fig. 2 - The four different types of super-elements used to model the lock gate

As a consequence, all the $N$ super-elements constituting the gate are characterized as being of type A, B, C or D and their individual resistances $P_{i}$ may be evaluated by an analytical formula. However, in order to evaluate $P_{l o c}$, the two following hypotheses are postulated:

- $\quad$ a super-element remains inactive $\left(P_{i}=0\right)$ as long as a geometrical contact is not established with the striking bow.

- the deformations taking place in a super-element do not affect the surrounding ones. Consequently, each element is supposed to be totally decoupled from the others.

With these assumptions, it transpires that the local resistance $P_{l o c}$ may be simply obtained by summing all the individual contributions coming from the $N$ super-elements, i.e.:

$$
P_{l o c}=\sum_{i=1}^{N} P_{i}(\delta)
$$

This last equation provides an estimation of the resistance associated to the localized crushing of the gate near the contact area. The next step is now to perform a similar derivation for the global deforming mode. 


\subsection{Global resistance}

The resistance $P_{g l o b}$ in the global mode is derived under the hypothesis that the gate is forced into an overall motion, where the bending effects are preponderant. This is roughly depicted in Fig. 3a, which shows the global deformation pattern in the case of a gate resting against a sill. During such a movement, it is clear that the main contribution to the resistance is coming from the horizontal girders and not really from the vertical frames. Indeed, the latter mainly follows the bending motion without dissipating a lot of energy, which justifies that their contribution can be neglected.

As a consequence, if the structure is reinforced by $M$ horizontal girders, it may be seen as a set of $M$ independant beams simply supported by the lock walls. These beams are bent in their plane and submitted to an individual displacement $\delta_{k}$ (Fig. 3b) that may be related to the ship penetration $\delta$ through simple analytical relations.

(a) Global bending motion of the gate

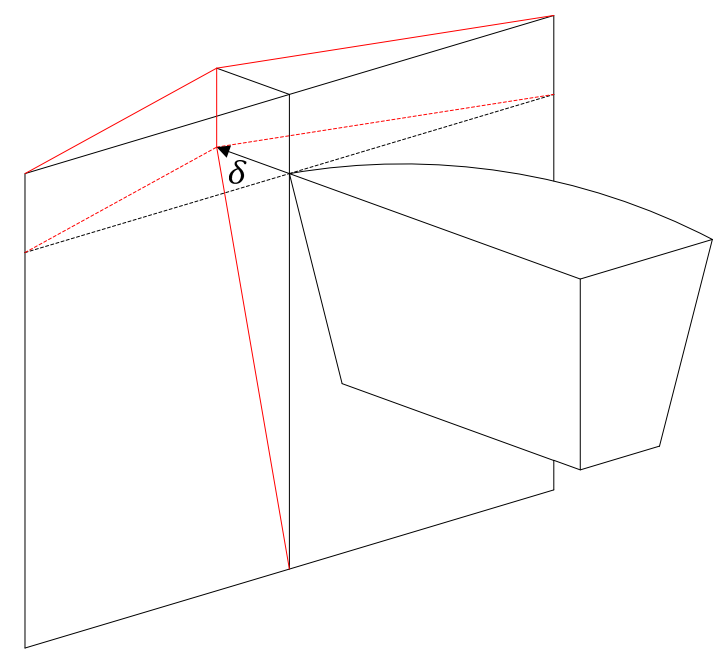

(b) Individual bending of the horizontal girders

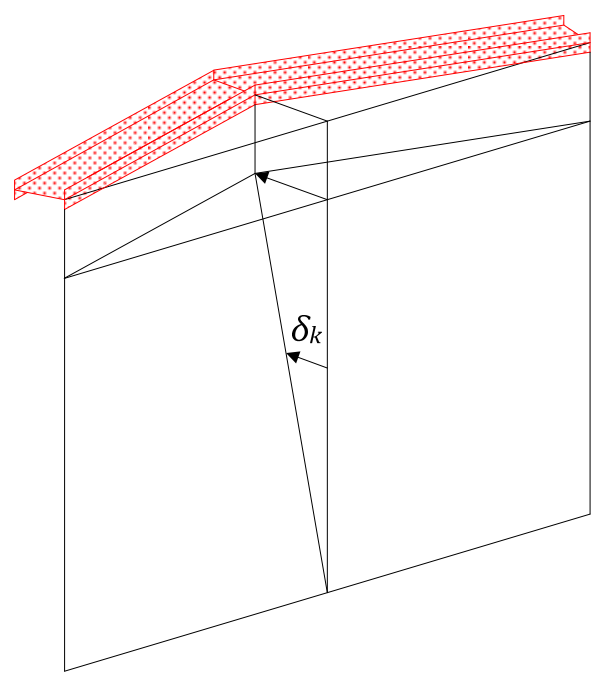

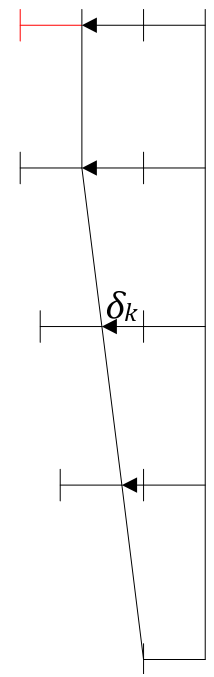

Fig. 3 - Mechanical model and displacement field for the global mode

Of course, during this overall motion, each of the $M$ girders opposes an individual resistance $P_{k}$, which can be simply evaluated by using the classical bending theory of beams in both the elastic and plastic regimes. For conciseness, the analytical derivation of $P_{k}$ will not be presented here but additional details may be found in [10]. Furthermore, as the $M$ horizontal girders are assumed to be independent, the global resistance $P_{g l o b}$ may be again calculated by summing all the contributions $P_{k}$. Doing so leads to:

$$
P_{\text {glob }}(\delta)=\sum_{k=1}^{M} P_{k}(\delta)
$$

So finally, it appears that $\boldsymbol{P}_{\boldsymbol{l o c}}$ and $\boldsymbol{P}_{\boldsymbol{g l o b}}$ can be evaluated by the closed-form expressions given in (3) and (4). These solutions may then be combined in accordance with equation (1) to find an approximation of the gate resistance $\boldsymbol{P}$.

\subsection{Results}

To validate the simplified analytical approach exposed here over, it is now applied to a given lock gate. The aim is to compare the analytical prediction $P(\delta)$ calculated with equation (1) to a numerical solution obtained by simulating the collision with the LS-DYNA software. To do so, a finite-element model of the striking vessel is first built with PATRAN. The latter is presented on Fig. 4a, where it can be seen that a finer mesh is used in the region located near the impact point 
in order to provide better contact conditions. The ship is assumed to have a total mass of 4000 tons and an initial velocity of $2 \mathrm{~m} / \mathrm{s}$.

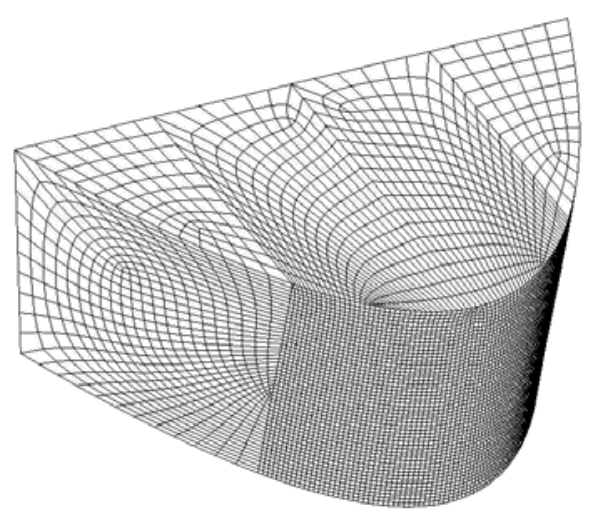

(a) Finite-element model of the vessel

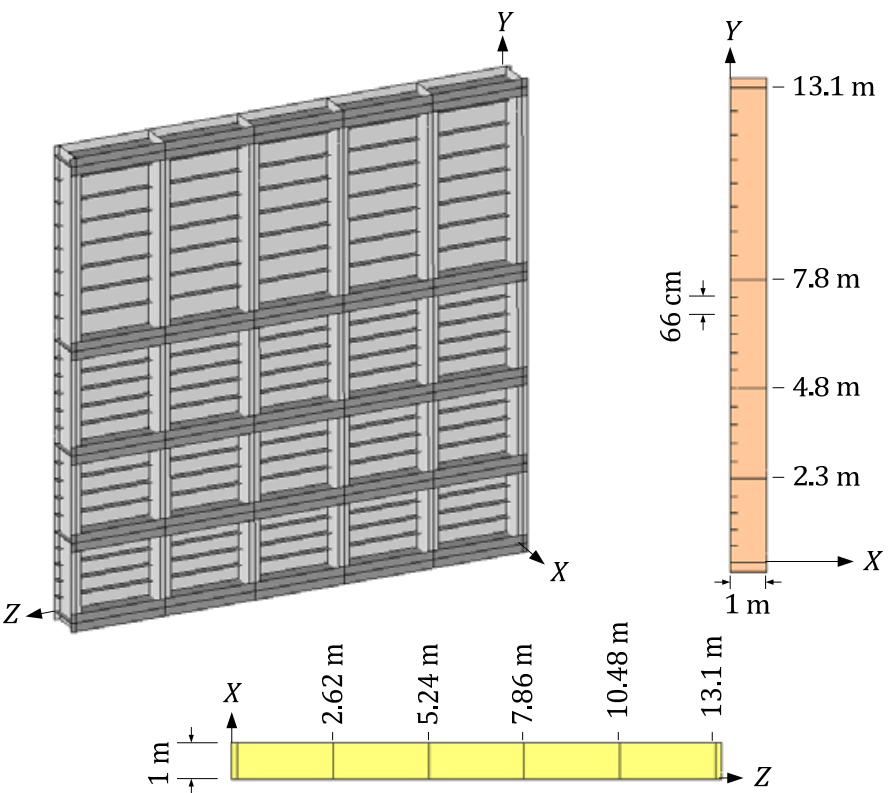

(b) Finite-element model of the gate

Fig. 4 - Finite-element models used for the numerical simulation [1]

The gate considered in this example is depicted in Fig. 4a with its main geometrical properties. It has a single plating, reinforced by 5 horizontal girders, 6 vertical frames and additional smaller stiffeners. It is made of mild steel, with a Young modulus of $210 \mathrm{GPa}$ and a yield stress of $235 \mathrm{MPa}$.

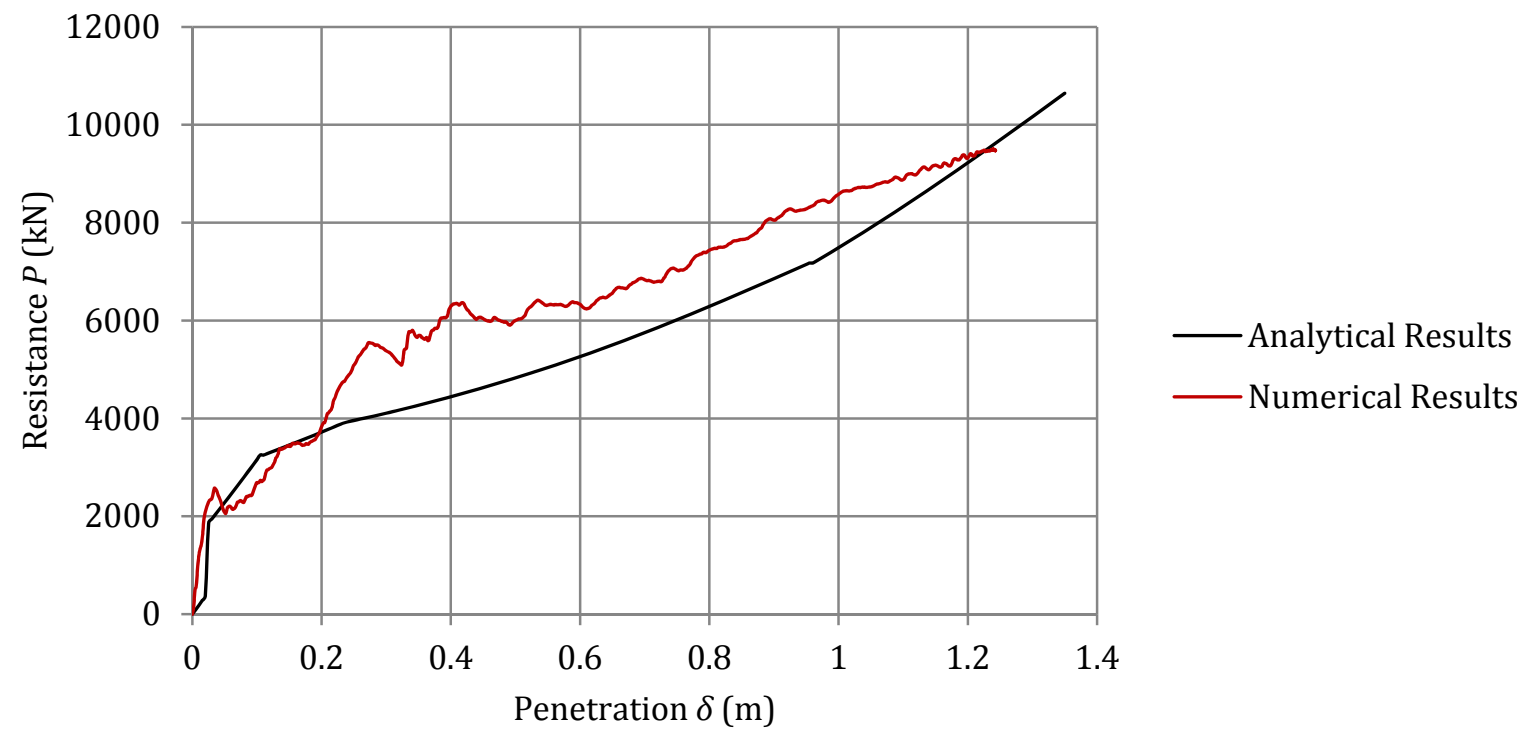

Fig. 5 - Comparison of the resistance curves obtained numerically and analytically [1]

The resistance obtained by the present simplified approach is compared to the numerical solution of LS-DYNA on Fig. 5. As it can be seen, the agreement between the two curves is satisfactory. Apart from the example presented in this paper, many other validations have been performed for both plane and mitre gates. In each case, the maximal penetration of the striking vessel predicted by the analytical method has been found to be conservative and in an acceptable agreement with the finite-element results. Such observations show that it is possible to quickly approximate the impact resistance of a lock gate without having to resort to numerical simulations. 


\section{Seismic design of lock gates}

During an earthquake, the lock chamber is submitted to a longitudinal ground acceleration that produces an additional hydrodynamic pressure directly applied on the gate. This latter is known to have the three following contributions:

- the convective pressure associated to the sloshing appearing at the free surface of the lock chamber. However, as lock chambers are usually quite long, this contribution is negligible.

- the rigid pressure, which is derived by assuming that the upstream and downstream gates are rigid and therefore simply follow the ground acceleration.

- the flexible pressure, which is coming from the own vibrations of the gates during the earthquake. These ones also produce an additional contribution that would not be present if the gate were perfectly rigid.

The two first parts have already been extensively investigated in the literature. Since the pioneer work of Westergaard [11], many other developments have been performed by Haroun [12], Housner [13], Ibrahim [14] or Epstein [15] to derive analytical formulae giving the rigid and convective pressures. Unfortunately, assessing the third contribution is more difficult because of the coupling between the structure and the fluid. In fact, the flexible pressure is directly influenced by the vibrations of the gate, which has also an influence on the gate displacements.

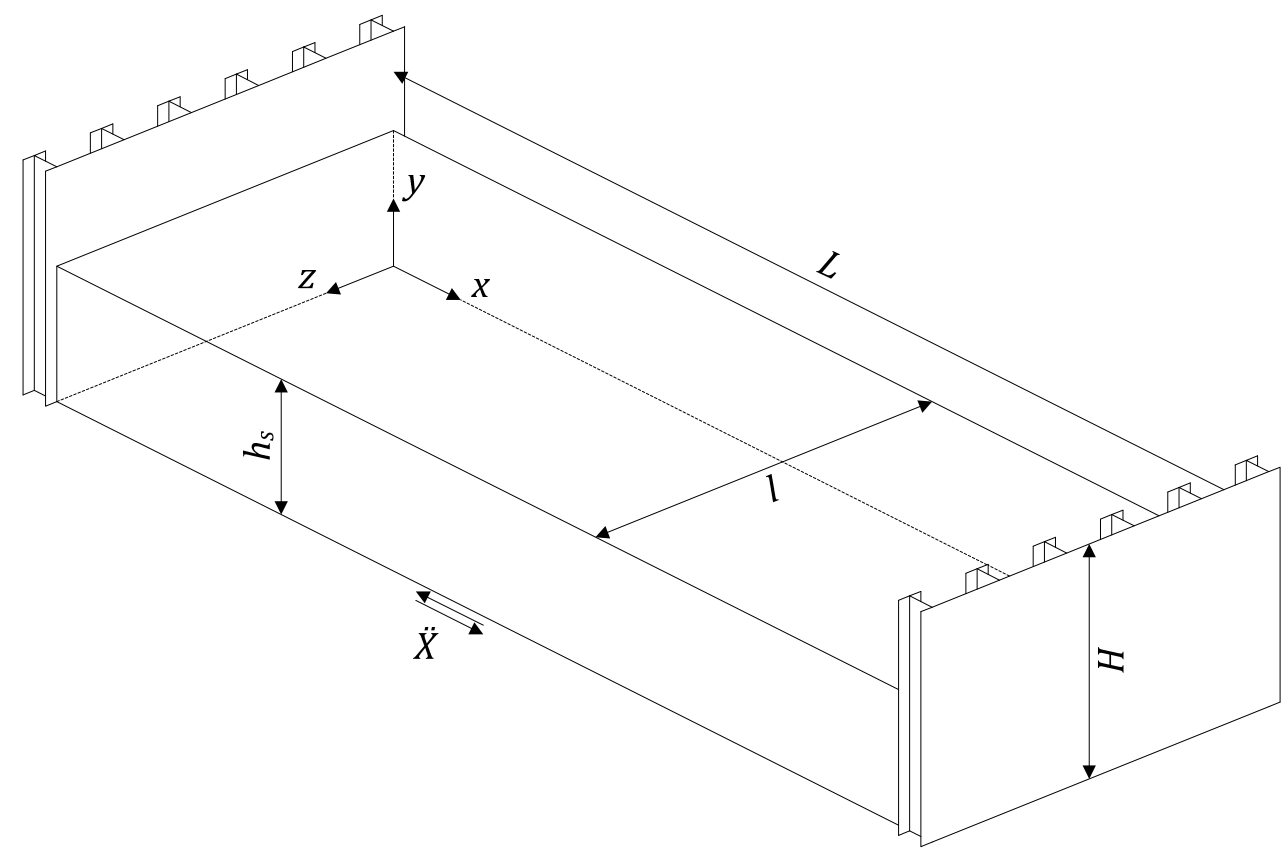

Fig. 6 - Main geometrical parameters of the lock chamber

The goal of this section is to investigate these phenomena precisely and to propose a simplified analytical method to approximate quickly the total hydrodynamic pressure. The derivation is focused on structures having a single plating, reinforced by horizontal girders and vertical frames. The width, depth and length of the lock chamber are denoted by $l, h_{s}$ and $L$ respectively, as depicted in Fig. 6. Regarding the boundary conditions, the gates are supposed to be simply supported at the lock walls, but they are free at the bottom.

\subsection{Free vibration analysis of the dry gate}

As a first step, the aim is to approximate analytically the natural frequencies $\omega_{i}$ and modes shapes $\delta_{i}$ characterizing the dry gate. This can be achieved by applying the Rayleigh-Ritz method, as described by Shames [16]. To do so, the basic idea is to decompose the mode shapes 
$\delta_{i}$ as a set of admissible function $\psi_{j}$ that may be arbitrarily chosen, provided that they respect the boundary conditions. With this assumption, one may write:

$$
\delta_{i}(y, z)=\sum_{j=1}^{M} v_{j i} \psi_{j}(y, z)
$$

where $M$ is the total number of functions used in the combination and $v_{j i}$ is the unknown coefficient that will be determined later. In this paper, it is suggested to choose $\psi_{j}$ in accordance with the following relation:

$$
\psi_{j}(y, z)=f_{j}(y) g_{j}(z)
$$

in which $f_{j}$ and $g_{j}$ characterize the vibrations of the main horizontal and vertical stiffening elements respectively. Furthermore, as the girders are simply supported in $z=0$ and $z=l$, it seems reasonable to use the eigenmodes of a doubly supported beam of span $l$ for $g_{j}$ (Fig. 7a). Similarly, as the gate is free at the top and bottom edges, the mode shapes of free-free beams can be conveniently chosen for $f_{j}$ (Fig. 7b). As the mathematical expressions of $f_{j}$ and $g_{j}$ can be easily found in the literature [17], they are not recalled here. Inserting them in equation (5) finally leads to an approximate decomposition of the eigenmodes characterizing the dry structure. The next step is then to determine the unknown coefficients $v_{j i}$.
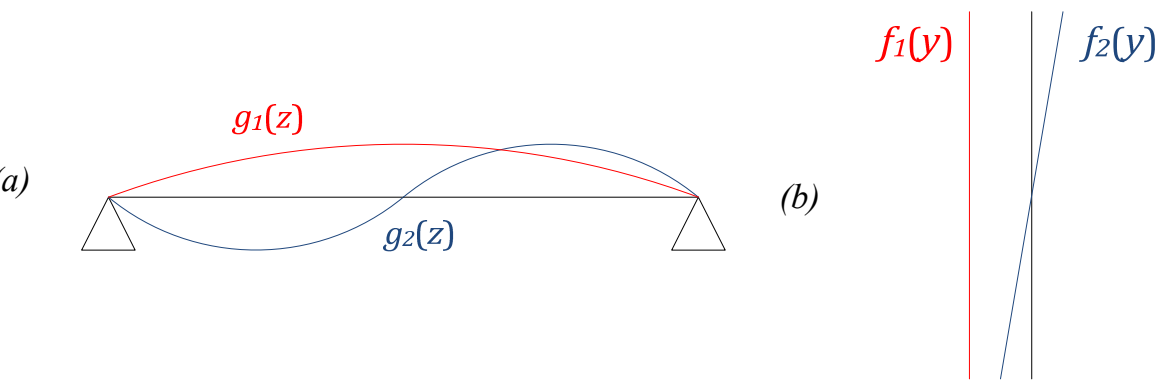

Fig. 7 - First eigenmodes of simply supported and free-free beams

In order to achieve this goal, the evaluation of the internal and kinetic energies $U$ and $T$ associated to the free vibrations of the structure is required. $U$ may be seen as the sum of two following contributions $U_{p}$ and $U_{s}$ coming from the plating and the stiffeners respectively:

$$
\begin{aligned}
& U_{p}=\frac{D}{2} \iint_{A}\left(\left(\frac{\partial^{2} \delta_{i}}{\partial y^{2}}\right)^{2}+\left(\frac{\partial^{2} \delta_{i}}{\partial z^{2}}\right)^{2}+2 v \frac{\partial^{2} \delta_{i}}{\partial y^{2}} \frac{\partial^{2} \delta_{i}}{\partial z^{2}}+2(1-v)\left(\frac{\partial^{2} \delta_{i}}{\partial y \partial z}\right)^{2}\right) d y d z \\
& U_{r}=\sum_{n} \frac{E I_{h, n}}{2} \int_{0}^{l}\left[\frac{\partial^{2} \delta_{i}}{\partial z^{2}}\left(y_{n}, z\right)\right]^{2} d z+\sum_{n} \frac{E I_{v, n}}{2} \int_{0}^{H}\left[\frac{\partial^{2} \delta_{i}}{\partial y^{2}}\left(y, z_{n}\right)\right]^{2} d y
\end{aligned}
$$

where $v$ and $E$ are the Poisson ratio and Young modulus respectively. The plate is characterized by its surface $A$ and bending flexibility $D$. The inertias of the horizontal and vertical reinforcing elements are designated by $I_{h, n}$ and $I_{v, n}$ respectively, while $y_{n}$ and $z_{n}$ denotes the discrete locations occupied by the frames and the girders along the vertical and horizontal axes (Fig. 6). Similarly, the kinetic energy $\omega_{i}^{2} T$ can be obtained by summing the two subsequent contributions $\omega_{i}^{2} T_{p}$ and $\omega_{i}^{2} T_{s}$ : 


$$
\begin{aligned}
& \omega_{i}^{2} T_{p}=\frac{\rho t_{p}}{2} \iint_{A} \delta_{i}^{2}(y, z) d y d z \\
& \omega_{i}^{2} T_{r}=\sum_{n} \frac{\rho A_{h, n}}{2} \int_{0}^{l} \delta_{i}^{2}\left(y_{n}, z\right) d z+\sum_{n} \frac{\rho A_{v, n}}{2} \int_{0}^{H} \delta_{i}^{2}\left(y, z_{n}\right) d y
\end{aligned}
$$

where $\rho$ is the mass density, while $A_{h, n}$ and $A_{v, n}$ denote the cross-sectional areas of the girders and the frames. Introducing (5) in equations (7) to (10), it is possible to get matrix formulations for $T$ and $U$. If $\boldsymbol{v}_{i}$ is the vector containing the unknown coefficients $v_{j i}$ mentioned in equation (5), it could be shown that [10]:

$$
T=\frac{1}{2} \sum_{j=1}^{M} \sum_{k=1}^{M} v_{j i} T j k v_{k i}=\frac{1}{2} \boldsymbol{v}_{i}^{T}[T] \boldsymbol{v}_{i} \quad ; \quad U=\frac{1}{2} \sum_{j=1}^{M} \sum_{k=1}^{M} v_{j i} U j k v_{k i}=\frac{1}{2} \boldsymbol{v}_{i}^{T}[U] \boldsymbol{v}_{i}
$$

Once the previous results are established, the Rayleigh-Ritz method requires to minimize the Rayleigh quotient $R$ to find the vectors $\boldsymbol{v}_{i}$. According to Shames [16], this is achieved by solving the following classical eigenvalue problem:

$$
\min \{R\}=\min \left\{\frac{\boldsymbol{v}_{i}^{T}[T] \boldsymbol{v}_{i}}{\boldsymbol{v}_{i}^{T}[U] \boldsymbol{v}_{i}}\right\} \Leftrightarrow \operatorname{det}\left([U]-\omega_{i}^{2}[T]\right)=0 ;\left([U]-\omega_{i}^{2}[T]\right) \boldsymbol{v}_{i}=\mathbf{0}
$$

Doing so leads to the vibration frequencies $\omega_{i}^{2}$ and the coefficients $v_{j i}$ that may be further introduced in (5) to get the dry modes shapes $\delta_{i}$.

\subsection{Dynamic analysis of the gate}

Once the modal properties of the gate are calculated, it is possible to perform a dynamic analysis in order to determine the total hydrodynamic pressure. To do so, the idea is to express the out-ofplane displacements $\boldsymbol{u}$ of the structure as a combination of the eigenmodes $\boldsymbol{\delta}_{\boldsymbol{i}}$ calculated previously. In fact, this approach is strictly similar to performing a classical modal decomposition. Consequently, if only the first $\boldsymbol{N}$ mode shapes are used in the decomposition, one may write:

$$
u(y, z, t)=\sum_{j=1}^{N} q_{j}(t) \delta_{j}(y, z) \Leftrightarrow \delta u(y, z, t)=\sum_{k=1}^{N} \delta q_{k}(t) \delta_{j}(y, z)
$$

where $q_{j}$ are unknown time coefficients that can be fixed by applying the virtual work principle. This theorem states that equating the internal and external virtual works for any kinematically admissible displacement field leads to a necessary and sufficient equilibrium condition for the structure. Consequently, the derivation of $q_{j}$ requires to evaluate:

- the internal work $\boldsymbol{\delta} \boldsymbol{W}_{\text {int }}$ associated to the deformations of the gate when this one is submitted to a virtual displacements field $\boldsymbol{\delta} \boldsymbol{u}$.

- the external work $\boldsymbol{\delta} \boldsymbol{W}_{\text {ext }}$ done by all the forces acting on the structure for the virtual displacements field $\boldsymbol{\delta} \boldsymbol{u}$.

As explained by Buldgen et al. [18], if $\boldsymbol{\delta} \boldsymbol{u}$ is calculated in accordance with equation (13), the derivation of $\boldsymbol{\delta} \boldsymbol{W}_{\text {int }}$ may be obtained by summing the virtual energies $\boldsymbol{\delta} \boldsymbol{W}_{\boldsymbol{p}}$ and $\boldsymbol{\delta} \boldsymbol{W}_{\boldsymbol{s}}$ associated to the plating and the stiffeners respectively. These ones may be shown to be as follows [10]: 


$$
\begin{gathered}
\delta W_{p}=D \int_{0}^{H} \int_{0}^{l}\left(\frac{\partial^{2} u}{\partial y^{2}} \frac{\partial^{2} \delta u}{\partial y^{2}}+\frac{\partial^{2} u}{\partial z^{2}} \frac{\partial^{2} \delta u}{\partial z^{2}}+v \frac{\partial^{2} u}{\partial y^{2}} \frac{\partial^{2} \delta u}{\partial z^{2}}+v \frac{\partial^{2} \delta u}{\partial y^{2}} \frac{\partial^{2} u}{\partial z^{2}}+2(1-v) \frac{\partial^{2} u}{\partial y \partial z} \frac{\partial^{2} \delta u}{\partial y \partial z}\right) d y d z \\
\delta W_{r}=\sum_{n} E I_{h, n}\left[\int_{0}^{l} \frac{\partial^{2} u}{\partial z^{2}} \frac{\partial^{2} \delta u}{\partial z^{2}} d z\right]_{y=y_{n}}+\sum_{n} E I_{v, n}\left[\int_{0}^{H} \frac{\partial^{2} u}{\partial y^{2}} \frac{\partial^{2} \delta u}{\partial y^{2}} d y\right]_{z=z_{n}}
\end{gathered}
$$

Similarly, the evaluation of $\boldsymbol{\delta} \boldsymbol{W}_{\text {ext }}$ may also be achieved by adding the contributions coming from the external forces applied on the gate. Only the following ones are considered here:

- The inertia forces due to the acceleration $\ddot{\boldsymbol{u}}+\ddot{\boldsymbol{X}}$ and acting on both the plating and the stiffening system. During the earthquake, they produce the following external work:

$$
-\int_{0}^{H} \int_{0}^{l} \rho t_{p} \delta u(\ddot{u}+\ddot{X}) d y d z-\sum_{n} \rho A_{h, n}\left[\int_{0}^{l} \delta u(\ddot{u}+\ddot{X}) d z\right]_{y=y_{n}}-\sum_{n} \rho A_{v, n}\left[\int_{0}^{H} \delta u(\ddot{u}+\ddot{X}) d y\right]_{z=z_{n}}
$$

- The mass and stiffness damping forces. The latter may be directly expressed as a function of the mechanical and geometrical properties of the structure, but for convenience, they are simply denoted here by $\boldsymbol{f}_{\boldsymbol{d}}$. More details about their analytical derivation can be found in references [10] and [16]. As $\boldsymbol{f}_{\boldsymbol{d}}$ is a force per unit of area, the corresponding external work is:

$$
-\int_{0}^{H} \int_{0}^{l} f_{d}(y, z, t) \delta u(y, z, t) d y d z
$$

- The pressure forces produced by the water surrounding the gate that are the sum of the convective, rigid and flexible terms. Considering only these two latter contributions and denoting them by $\boldsymbol{p}_{\boldsymbol{r}}$ and $\boldsymbol{p}_{\boldsymbol{f}}$ respectively, the following expression can be established for the corresponding external work:

$$
-\int_{0}^{h_{s}} \int_{0}^{l}\left(p_{r}(y, t)+p_{f}(y, z, t)\right) \delta u(y, z, t) d y d z
$$

As detailed by Kim et al. [19], it is worth mentioning that $\boldsymbol{p}_{\boldsymbol{r}}$ is directly related to the seismic accelaration $\ddot{\boldsymbol{X}}$, while the time evolution of $\boldsymbol{p}_{\boldsymbol{f}}$ is rather influenced by the accelerations $\ddot{\boldsymbol{u}}$ of the gate.

For all the previous equations, a modal extension can be obtained by using the decomposition (13) expressing $\boldsymbol{u}$ and $\boldsymbol{\delta} \boldsymbol{u}$ as a combination of the eigenmodes. Doing so, it is possible to show that [10]:

$$
\delta W_{i n t}=\sum_{k=1}^{N} \delta q_{k} \sum_{j=1}^{N} q_{j} K_{j k} \quad ; \quad \delta W_{e x t}=-\sum_{k=1}^{N} \delta q_{k}\left[\sum_{j=1}^{N}\left(\ddot{q}_{j} M_{j k}+q_{j}\left(\alpha M_{j k}+\beta K_{j k}\right)-\ddot{q}_{j} J_{j k}\right)-P_{k} \ddot{X}\right]
$$

where $\boldsymbol{\alpha}$ and $\boldsymbol{\beta}$ are the Rayleigh coefficients used for the mass and stiffness proportional damping. In these two expressions, it is also worth mentioning that $\boldsymbol{M}_{\boldsymbol{j} \boldsymbol{k}}$ and $\boldsymbol{K}_{\boldsymbol{j} \boldsymbol{k}}$ translates the effects of the mass and the stiffness of the gate, while $\boldsymbol{J}_{\boldsymbol{j} \boldsymbol{k}}$ and $\boldsymbol{P}_{\boldsymbol{k}}$ are related to the flexible and rigid pressures. Of course, all these parameters may be evaluated analytically [10], but for conciseness, the corresponding detailed relations are not provided here. 
Finally, in accordance with the virtual work principle, $\boldsymbol{\delta} \boldsymbol{W}_{\text {int }}$ and $\boldsymbol{\delta} \boldsymbol{W}_{\text {ext }}$ may be equated to get a set of $\boldsymbol{N}$ equations that may be written in the following matrix form:

$$
([M]+[J]) \ddot{q}(t)+(\alpha[M]+\beta[K]) \dot{q}(t)+[K] q(t)=P \ddot{X}(t)
$$

in which $\boldsymbol{q}$ is a vector containing the unknown coefficients $\boldsymbol{q}_{\boldsymbol{j}}$. For a given accelerogram $\ddot{\boldsymbol{X}}$, the latter can be easily obtained by solving (20) with the help of the Newmark integration scheme for example. Once the parameters $\boldsymbol{q}_{\boldsymbol{j}}$ are determined, they can be inserted in (13) to get the displacement and acceleration $\ddot{\boldsymbol{u}}$, from which it is possible to get the flexible pressure $\boldsymbol{p}_{\boldsymbol{f}}$.

As a final comment, it should be mentioned that a careful examination of (20) shows that this equation is quite similar to the classical relation expressing the dynamic equilibrium of a structure submitted to the external forces $\boldsymbol{P} \ddot{\boldsymbol{X}}$. Nevertheless, this is not exactly the case of the matrix [J] that is added in the left hand side of (20). This one translates the effect of the surrounding water and therefore denotes the fluid-structure interaction. However, if the structure were perfectly rigid, this coupling would not be present as $[\boldsymbol{J}]=[\mathbf{0}]$ in this case. With such an assumption, (20) would be strictly equivalent to the equilibrium equation that is considered in the added-mass method as applied by Forsyth and Porteous [20] for example.

\subsection{Results}

In order to corroborate the results provided by the analytical method exposed above, the gate presented in section 2.4 is now used in a validation process, but two differences regarding the finite-element modeling should however be mentioned:

- the material law is supposed to be perfectly linear elastic, with a Young modulus of $210 \mathrm{GPa}$. The mass density and the Poisson ratio are equal to $7850 \mathrm{~kg} / \mathrm{m}^{3}$ and 0.3 respectively. With such properties, no plasticity is allowed during the numerical simulation.

- Unlike the finite-element model presented on Fig. 4, the horizontal girders, the vertical frames and the stiffeners are no longer modeled with shell elements. Beam elements are used now in order to slightly reduce the meshing effort.

As a first step, the f proposal is to check if the analytical derivation of the modal properties described in section 3.1 leads to a reasonable estimation. To do so, the vibration frequencies and mode shapes are computed by using the NASTRAN software. As expected for this kind of stiffened structure, the numerical results point out that the gate has only two dominant modes and a lot of local ones. Of course, the latter cannot be captured by the simplified procedure detailed in section 3.1 because of the generating functions $\psi_{j}$ chosen in equation (5) to approximate the eigenmodes. Consequently, it is only required to check here if there is a satisfactory agreement for the main global modes.

Table 1

Comparison of the eigenfrequencies

\begin{tabular}{|c|c|c|c|c|c|}
\hline \multirow{3}{*}{ Mode } & \multicolumn{3}{|c|}{ Frequency $(\mathrm{Hz})$} & \multicolumn{2}{c|}{ Error (\%) } \\
\cline { 2 - 6 } & NASTRAN & $\begin{array}{c}\text { LS- } \\
\text { DYNA }\end{array}$ & Analytical & NASTRAN & $\begin{array}{c}\text { LS- } \\
\text { DYNA }\end{array}$ \\
\hline 1 & 19.2 & 20.0 & 21.3 & 10.9 & 6.5 \\
\hline 2 & 23.3 & 25.3 & 26.0 & 11.6 & 2.7 \\
\hline
\end{tabular}

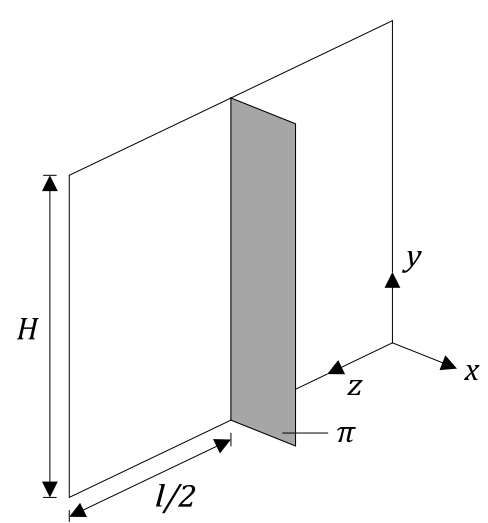

Fig. 8 - Definition of plane $\pi$ 
The natural frequencies derived numerically and analytically are listed in Table 1 . An estimation computed by the LS-DYNA software is also presented. From these results, it appears that the maximal error is limited to $7 \%$, which is quite satisfactory for a pre-design stage.

In order to check if there is also a sufficient agreement on the eigenmodes, one can compare the mode shapes in the vertical plane $\pi$ that is perpendicular to the gate and crossing it in the middle (i.e. in $z=l / 2$ ), as depicted on Fig. 8. The corresponding results are depicted on Fig. 9 for the two main modes. From these curves, it can be concluded that there is a quite good correlation between the analytical and numerical solutions.

Once the modal properties of the dry structure are validated, the next step is now to check if the hydrodynamic pressure calculated in section 3.2 provides a reasonable estimation of the numerical solutions. To do so, the previous gate is supposed to be in contact with a chamber having a length $L$ of $100 \mathrm{~m}$ and filled with water up to a level $h_{s}$ of $8 \mathrm{~m}$ (Fig. 6). The lock is submitted to the longitudinal seismic acceleration $\ddot{X}$ of Fig. 10 .
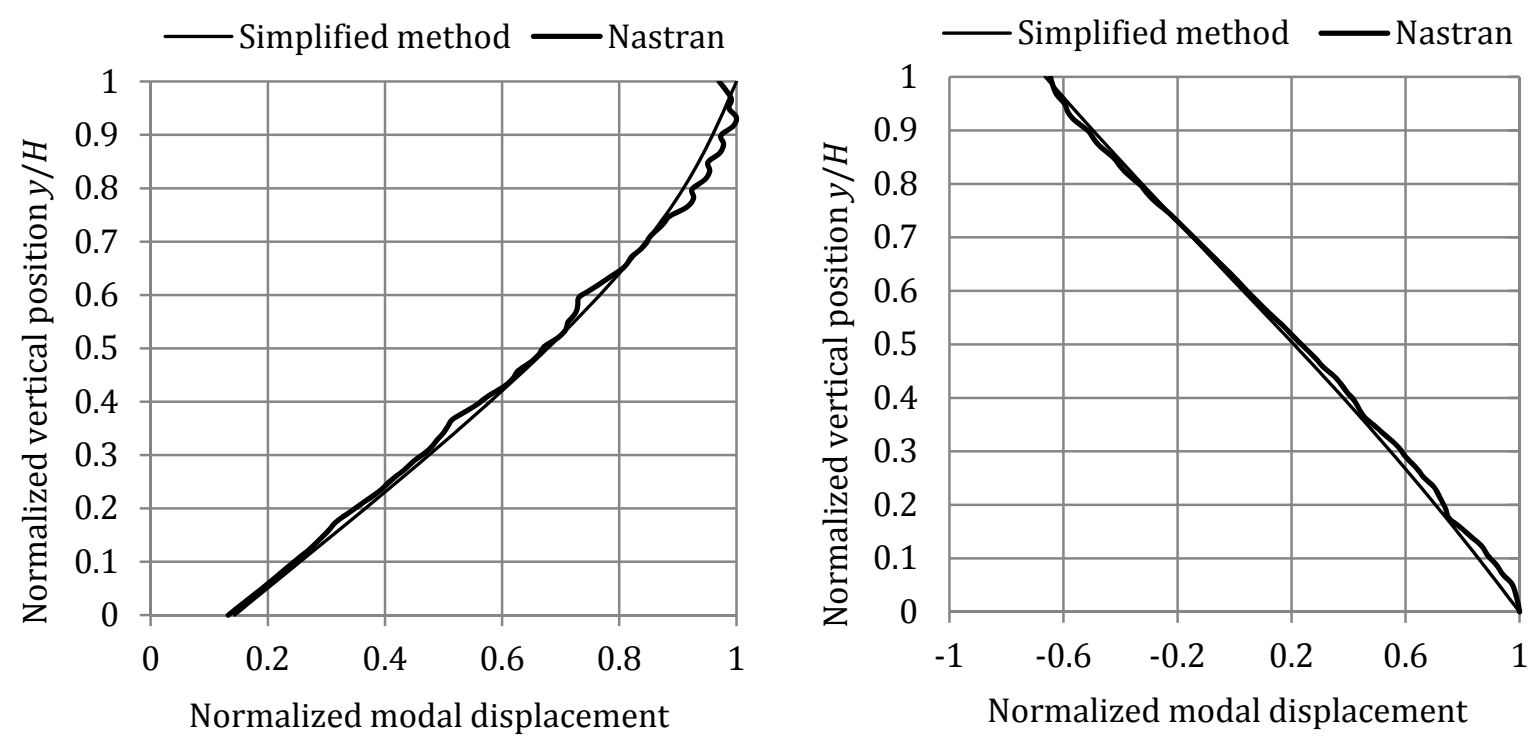

Fig. 9 - Comparison of the first and second eigenmodes obtained numerically and analytically

The numerical simulation is performed using the LS-DYNA software. In order to simulate the interaction between the gate and the water, it is required to have a finite elements model of both of them. In this paper, constant stress solid elements [21] are used for the liquid, which means that the water is considered as an elastic medium with no shearing. The mesh of the fluid domain is regular, with a size of $20 \times 20 \times 20 \mathrm{~cm}$. Regarding the structure, as depicted on Fig. 6, the upstream and downstream gates are assumed to be the same as the one presented in section 2.4. The plating is model with Belytscko-Tsay shell elements [21], while Hughes-Liu beams [21] are used for the stiffening components.

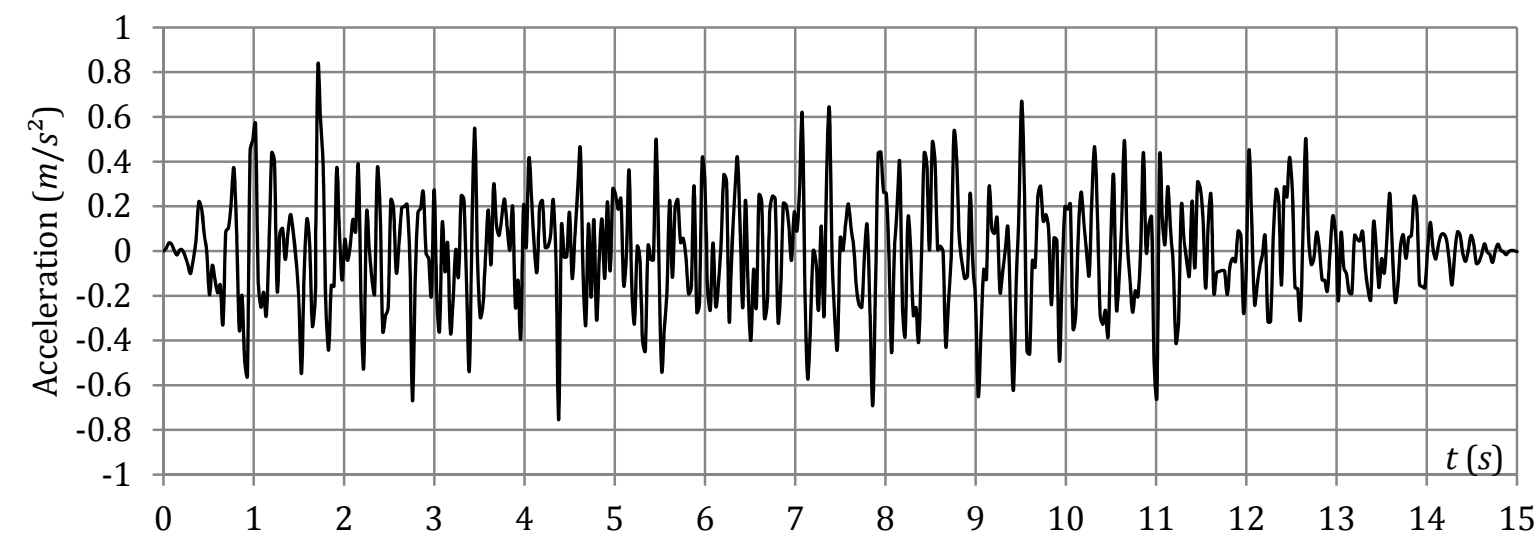

Fig. 10 - Accelerogram used for the numerical simulations 
In order to prevent the fluid from passing across the gates, the LS-DYNA contact algorithm is used, which means that distinct nodes are defined for the structure and the fluid. Consequently, the liquid is free to slide over the gate without friction. Similarly, in order to avoid any leakage at the bottom and at the lock walls, the displacements of the fluid are adequately prohibited by appropriated boundary conditions at these locations.

In order to check if the formulae derived in section 3.2 provide a sufficient approximation, the total resulting hydrodynamic pressure force $F$ applied on the structure is proposed here to be compared. This one is simply obtained by integrating the rigid and flexible contributions $p_{r}$ and $p_{f}$ over the entire surface of the gate, i.e.:

$$
F(t)=\int_{0}^{h_{s}} \int_{0}^{l}\left(p_{r}(y, t)+p_{f}(y, t)\right) d y d z
$$

The time evolutions of $F$ predicted analytically and calculated by LS-DYNA are plotted on Fig. 11, for the seismic accelaration of Fig. 10, from which it can be concluded that there is a quite good agreement between the two curves.
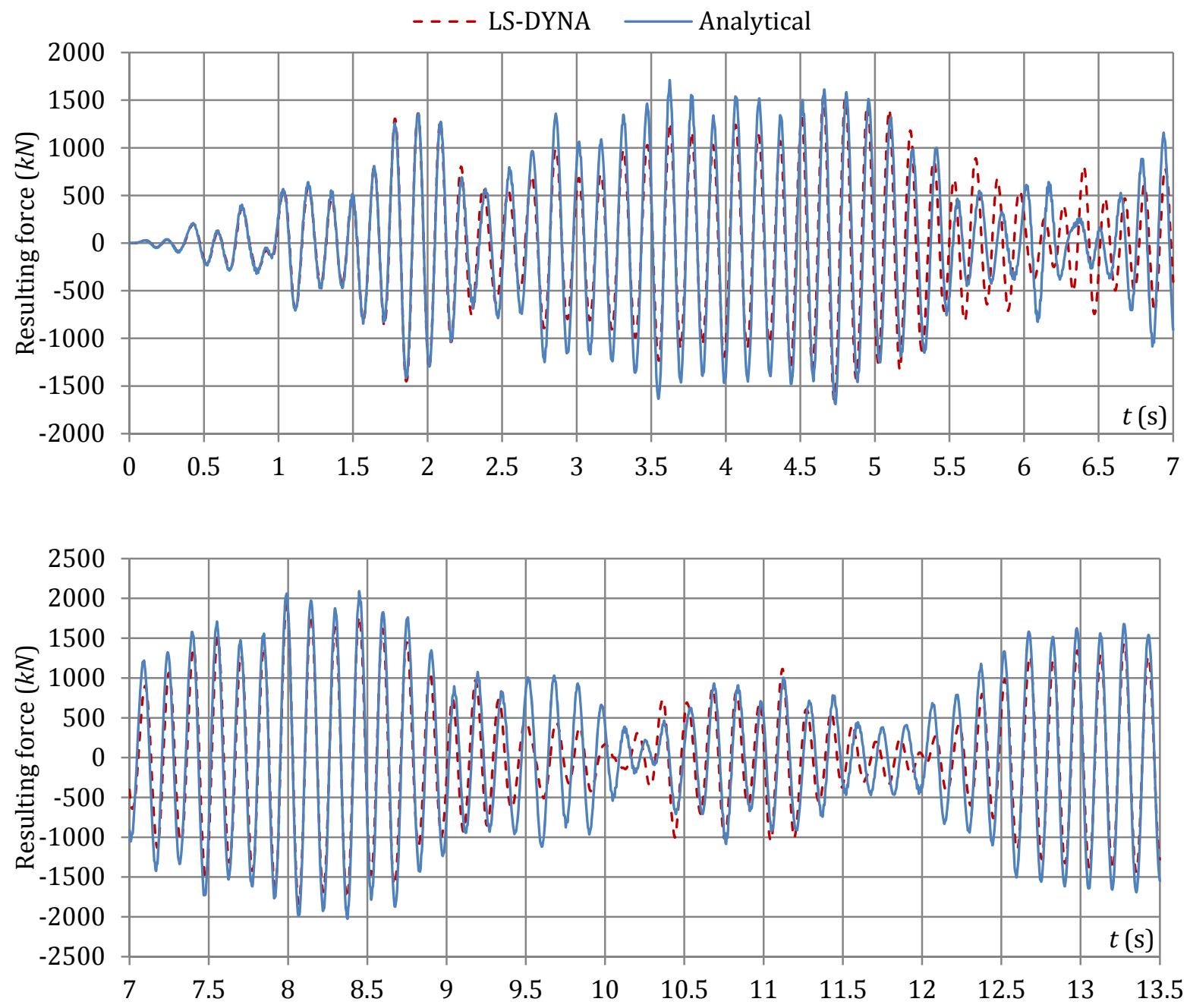

Fig. 11 - Time evolution of the total hydrodynamic force applied on the gate

From the results presented on Fig. 11, it can be also observed that the present simplified method appears to be conservative as it tends to overestimate the peak values. In order to have a better comparison, the latter are reported in Table 2, where the analytical prediction $\left(F_{T}\right)$ is seen to overestimate the extreme positive and negative forces $\left(F_{N}\right)$ by an amount of $10 \%$ more or less. 
Extreme values of the resulting pressure force

\begin{tabular}{|l|c|c|c|c|c|}
\cline { 2 - 6 } \multicolumn{1}{c|}{} & $\begin{array}{c}\text { Analytical } \\
\text { solution } F_{T}\end{array}$ & $\begin{array}{c}\text { Numerical } \\
\text { solution } F_{N}\end{array}$ & $\begin{array}{c}\text { Rigid } \\
\text { solution } F_{R}\end{array}$ & $\begin{array}{c}\text { Relative difference } \\
\left|1-F_{T} / F_{N}\right|\end{array}$ & $\begin{array}{c}\text { Ratio } \\
F_{N} / F_{R}\end{array}$ \\
\hline Maximal value & $2085.4 k N$ & $1936.1 k N$ & $375.2 k N$ & $7.7 \%$ & 3.2 \\
\hline
\end{tabular}

Another point that is interesting to investigate is the importance of accounting for the gate flexibility when evaluating pressure. In the case of a perfectly stiff structure, only the rigid contribution would be applied on it, leading to a total hydrodynamic force denoted by $F_{R}$. The ratios between the extreme values calculated by LS-DYNA for both a flexible $\left(F_{N}\right)$ and a rigid $\left(F_{R}\right)$ gate are also listed in the rightmost column of Table 2. From these results, it can be observed that assuming a perfectly rigid gate leads to a maximal resulting force five times smaller. Similarly, the minimal value is underestimated by a factor four. Of course, even at a pre-design stage, working with such approximations is not acceptable and point outs the necessity to account for the gate flexibility to correctly evaluate the total hydrodynamic pressure during a seism.

\section{Conclusion}

In this paper, two simplified analytical methods are briefly exposed to investigate lock gates submitted to two different accidental loads. The focus is first made on the resistance of such structures when they are impacted by a vessel. In this situation, the analytical derivation of the resistance is based on the idea that the gate exhibits two different successive behaviors. When the penetration is still quite small, a local deforming mode is postulated, which means that there is only a localized crushing of some components near the impact point. However, when the ship is moving forward, there is a switch from this local behavior to a global one, involving an overall bending of the entire gate.

During the first phase, the analytical derivation is based on the super-element method. To do so, the structure is divided into large entities, each of them being characterized by a closed-form relation giving its individual contribution to the local resistance. This one is then simply obtained by considering all the activated super-elements. On the other hand, during the second phase, the gate is assimilated to a set of horizontal beams that are simply bent between the two lock walls. Doing so provides an estimation of the global resistance. As a matter of validation, the results provided by the simplified method are compared to numerical solutions obtained by the LS-DYNA software, from which a quite satisfactory agreement is observed.

The second accidental load considered in this paper is the seismic event. For this situation, an analytical method is presented to quickly evaluate the total hydrodynamic pressure applied on the gate, due account being taken of the fluid-structure interaction. To do so, the modal properties of the dry gate are first estimated with the help of the Rayleigh-Ritz methods, in which beam eigenmodes are used as generating functions. Once the vibration frequencies and the mode shapes are extracted, they are used to perform a simplified dynamic analysis of the structure surrounded by water. These developments are then based on the virtual work principle. Here again, numerical simulations were performed with the NASTRAN and LS-DYNA finite-element software in order to corroborate the analytical solutions. A sufficient agreement can be observed from these comparisons. 
As a final comment, it is worth recalling that the goal of this paper is not to present simplified approaches that aim to substitute for finite-element methods. Indeed, the solutions exposed here above are not sufficiently detailed for a final design stage. However, they can be advantageously used during the conception of a lock gate, when it is not highly necessary to have very accurate estimations of these accidental actions. Indeed, the two procedures introduced in sections 2 and 3 do not require to build numerical models and run simulations, which means that they are not particularly time-expensive. This property is of course quite interesting during the pre-design stage.

\section{Acknowledgements}

The authors would like to thank the MSC Software Corporation for its support with the finiteelement software MSC PATRAN/NASTRAN that was used in many steps of this research. MSC PATRAN has been utilized as a powerful pre-processor for building the models required to study ship impacts and earthquakes on lock gates. On the other hand, MSC NASTRAN was used for the seismic simulations and to extract the modal properties of the structures studied in this paper.

\section{References}

[1]. Buldgen L., Le Sourne H., Rigo P. (2012). Simplified analytical method for estimating the resistance of lock gates to ship impacts, Journal of Applied Mathematics, Vol. 2012, 2012, pp. 1-39.

[2]. Buldgen L., Le Sourne H., Rigo P. (2013). Fast strength assessment of mitre gates to ship impact, International Journal of Crashworthiness, Vol. 18, pp. 1-21.

[3]. Le Sourne H., Rodet J.C., Clanet C. (2002). Crashworthiness analysis of a lock gate impacted by two river ships, International Journal of Crashworthiness, Vol. 7, pp. 371-396.

[4]. Buldgen L., Le Sourne H., Rigo, P. 2013. A simplified procedure to assess the strength of a ship impacting a lock miter gate, Proceedings of the ASME 2013 32nd International Conference on Ocean, Offshore and Arctic Engineering.

[5]. Zhang S.M. (1999). The mechanics of ship collisions, PhD Thesis, Department of Naval Architecture and Offshore Engineering, Technical University of Denmark.

[6]. Hong L., Amdahl J. (2008). Crushing resistance of web girders in ship collision and grounding, Marine Structures, Vol. 21, pp. 374-401.

[7]. Simonsen B.C. (1998). Ship grounding on rock - I. Theory, Marine Structures, Vol. 10, pp. 519-562.

[8]. Simonsen B.C., Ocakli H. (1999). Experiments and theory on deck girder crushing, Thin-Walled Structures, Vol. 34, pp. 195-216.

[9]. Amdahl J. (1983). Energy Absorption in ship-platform impact, PhD Thesis, Department of Marine Technology, Norwegian University of Science and Technology.

[10]. Buldgen L. (2015), Simplified analytical methods for the crashworthiness and the seismic design of lock gates, PhD Thesis, Structural Engineering Department, University of Liège.

[11]. Westergaard H.M. (1933). Water pressure on dams during earthquakes, Transactions of the American Society of Civil Engineers, Vol. 98, pp. 418-433.

[12]. Haroun M.A. (1984). Stress analysis of rectangular walls under seismically induced hydrodynamic loads, Bulletin of Seismological Society of America, Vol. 74, pp. 1031-1041.

[13]. Housner G.W. (1974). Dynamic pressures on accelerated fluid containers, Bulletin of Seismological Society of America, Vol. 47, pp. 15-37.

[14]. Ibrahim R.A. (2005). Liquid sloshing dynamics: theory and applications, Cambridge University Press.

[15]. Epstein H.I. (1976). Seismic design of liquid storage tanks, Journal of the Structural Division, Vol. 102, pp. 16591673.

[16]. Shames I.H. \& Dym C.L. (1995). Energy and finite element methods in structural mechanics, New Age International.

[17]. Leissa A.W. (1973). The free vibration of rectangular plates, Journal of Sound and Vibration, Vol. 31, pp. $257-293$.

[18]. Buldgen L., Gazerzadeh A., Bela A., Rigo P., Le Sourne H. (2014). A simplified procedure to assess the dynamic pressures on lock gates, Proceedings of the ICTWS 2014 7th Conference on Thin-Walled Structures.

[19]. Kim J.K., Koh K.H., Kwahk I.J. (1996). Dynamic response of rectangular flexible fluid containers, Journal of Engineering Mechanics, Vol. 122, pp. 807-817.

[20]. Forsyth G., Porteous A. (2000). The design and construction of seismically qualified steel caissons at Rosyth Royal Dockyard, The Structural Engineer, Vol. 78, pp. 24-31.

[21]. Hallquist J.O. (2006). LS-DYNA theoretical manual, Livermore Software Technology Corporation. 